\title{
BESOINS COMMUNICATIFS EN L1 COMME SOURCE D'ERREURS SOCIOLINGUISTIQUES EN LE CHEZ LES ÉTUDIANTS DÉBUTANTS EN PHILOLOGIE ROMANE
}

\begin{abstract}
Zając Jolanta, Besoins communicatifs en L1 comme source d'erreurs sociolinguistiques en LE chez les étudiants débutants en philologie romane [Communicative needs in L1 as a source of sociolinguistic mistakes in foreign language among beginning students of Romance philology], Studia Romanica Posnaniensia, Adam Mickiewicz University Press, Poznań, vol. XXXVIII/2: 2011, pp. 5-17. ISBN 978-83-232-2335-1. ISSN 0137-2475. DOI 10.2478/v10123-011-0010-1.
\end{abstract}

Speaking foreign language does not imply only mastering its lexical and morphosyntactic code, but, more importantly, learning to conceptualize differently the world around us. One of major sources of deviations in L2 is due to a very poor mastery of conventional structures (formulaic competence), an incorrect linguistic profiling and the confusion between the synthetic and analytical character of the L1 and the L2 (here Polish and French). Beginners in Romance philology often fall into a trap when trying to verbalize in a written form their communicative intentions. We propose here to analyze their sociolinguistic and sociocultural mistakes in order to imagine a course of remedial teaching.

« Je ne peux pas attendre que tu vas voir mon appartement et rencontrer mes amis ! (S16, corpus) - voilà l'exemple d'une phrase écrite par un des étudiants des groupes « débutants » en philologie romane lors de l'épreuve de mi-parcours (après un semestre d'études, environ 200 heures). Si nous la citons c'est pour mieux illustrer l'objectif de notre analyse qui consiste à nous interroger sur la nature de ce type d'écarts linguistiques, leurs sources et d'éventuelles démarches de remédiation. En effet, il ne s'agit pas de voir dans ces énoncés uniquement des déviations par rapport à la norme morphosyntaxique ou lexicale mais d'y repérer des défaillances sur le plan de l'expression calqué sur les langues que l'apprenant maîtrise mieux que le français et auxquelles il a recours pour assouvir ses besoins communicatifs.

\section{LA CONCEPTUALISATION DU MONDE ET SON EXPRESSION LANGAGIÈRE}

Le signe linguistique est arbitraire et immotivé, répétons après le linguiste génévois ou, si l'on préfère, les relations entre la forme du contenu et la substance du contenu et la forme de 1 'expression et la substance de l'expression ne sont pas iso- 
morphes. Les langues « structurent » le monde chacune à sa manière et laissent à leurs usagers la conviction, tout aussi erronée que profonde, qu'il n'y a qu'une manière juste de le percevoir, celle de la langue première le plus souvent. Notre vision du monde se construit ainsi à partir des concepts ou, mieux, catégories de concepts qu'une langue permet d'expliciter. Il s'ensuit que :

Le monde n'est donc pas une réalité objective existant en et de par elle-même. Il nous apparaît toujours d'une façon ou d'une autre par le biais de notre activité qui consiste à catégoriser sur la base de notre perception, de nos connaissances, de notre état d'esprit, bref de notre condition humaine (Delbecque (éd.), $2006: 34$ ).

Ainsi la présence d'un « conceptualisateur » (Delbecque (éd.), 2006) assure le lien incontournable entre les catégories conceptuelles d'une part et les signes linguistiques de l'autre. Faut-il pour autant voir dans la langue maternelle un élément inhibiteur dans l'acquisition d'une L2 ? Peut-on réellement constater avec autant de force que l'a fait Charles Bouton (1974 : 168) en affirmant que « la situation d'acquisition détermine un conflit immédiat entre les deux codes : celui de la langue maternelle, celui de la langue seconde. La formule verbale de la langue seconde ne se charge de signification qu'à travers l'écran de la langue maternelle, ce qui réduit l'action des lois de contiguïté ou d'association »?

C'est la notion d'interlangue qui vient à l'appui de la réponse négative à une question formulée de cette façon. En effet, nous avons là un concept qui éclaircit ce rapport jusque-là vu uniquement sous l'angle de la domination de la L1 face à la L2. L'interlangue serait le « système linguistique interne, individuel d'un apprenant « (Cuq, 2003 : 140) construit à partir des traits de la langue cible et de la langue source sans être néanmoins un simple mélange ou addition de l'une et de l'autre. Cette « compétence transitoire» (Corder, 1967), du fait d'être transitoire, est instable - quoique sujette à des fossilisations - et débouche sur toute sorte d'écarts, déviations et d'erreurs qui sont la conséquence naturelle du travail mental de l'apprenant en voie de structuration du système de la L2.

L'interlangue reste un concept abstrait, en revanche, au niveau de l'expression elle se laisse percevoir à travers de l'interparole (Albert et Py, 1986), cette dernière étant la manifestation et l'activation de l'interlangue. C'est l'interparole de chaque étudiant justement que nous pouvons saisir dans leurs productions écrites. C'est le lieu de la manifestation de leur interlangue, le résultat de leur conceptualisation du monde en L2, c'est alors que l'on se rend compte des problèmes dont la source n'est pas le code en soi mais une structuration conceptuelle du monde erronée par rapport à ce code.

\section{OỦ SONT LES FAUTES D’ANTAN?}

Les enseignants d'il y a quelques décennies étaient bien plus rassurés que nous aujourd'hui. Avec la notion de la faute surtout ils avaient la conscience tranquille dans 
leur travail, la faute doit être toujours supprimée, il faut la punir, il faut apprendre à l'éviter, elle incarne tout le mal dans le processus didactique. L'arrivée de l'erreur et avec elle ce qu'on a appelé « la pédagogie de l'erreur » a déjà pas mal perturbé cette simplicité initiale de la démarche didactique.

A commencer par une question toute banale, paraît-il, à laquelle la réponse ne l'est plus : mais qu'est-ce qu'une erreur ? (Marquilló Larruy, 2003 : 14). Peut-on prétendre à trouver une définition exhaustive, indépendamment des situations de production, du canal de la communication, du genre textuel activé ? Nous sommes d'accord avec R. Porquier et U. Frauenfelder (1980 : 17) quand ils affirment que « par rapport au système intermédiaire de l'apprenant on ne peut véritablement parler d'erreurs. On voit alors qu'il est impossible de donner de l'erreur une définition absolue. Ici comme en linguistique, c'est le point de vue qui définit l'objet ».

Certes, le $C E C R$ la définit aujourd'hui en termes d' " une déviation ou une représentation déformée de la compétence cible. Il s'agit alors d'une adéquation de la compétence et de la performance de l'apprenant qui a développé des règles différentes des normes de la L2 » (2001 : 118) mais nous sommes loin d'une définition bâtie autour de la notion de l'écart, centrale jadis dans ce type de discours.

L'évolution terminologique est d'ailleurs pertinente à voir a cette occasion et instructive si l'on réfléchit à la démarche didactique. Ainsi parler de la faute se relie à l'action de supprimer, éliminer, punir ; parler de l'erreur évoque plus « correction, pédagogie » et discuter des " marques transcodiques ou de répertoire verbal mixte » (Marquilló Larruy, 2003 : 61) renvoie vers une réflexion hétéronome ou autonome sur l'interlangue et l'interparole, leur évolution, leur adéquation aux contextes abordés.

Une conclusion en ressort, si bien formulée par le $C E C R$ : « les erreurs sont inévitables ; elles sont le produit transitoire du développement d'une interlangue par l'apprenant. Les fautes sont inévitables dans tout usage d'une langue, y compris par les locuteurs natifs » (2001: 118).

Tout élément du système linguistique et extralinguistique fournit des occasions multiples à recourir au "répertoire verbal mixte », ou plus simplement, à commettre des erreurs par un adepte d'une L2. Les niveaux morphologique, syntaxique, sémantique, phonétique, orthographique, pragmatique, sociolinguistique deviennent, chacun à son tour, celui qui va piéger la compétence communicative de l'apprenant. Il y a du pain sur la planche ! aurait-on envie de dire en classe de langue. Rien d'étonnant que le traitement de l'erreur nécessite de la patience et surtout de la persévérance, tant de la part de l'enseignant que de la part de l'apprenant, agacé à la fin par le nombre d'erreurs commises dans une moindre production en L2.

Nous sommes intéressée ici par un type particulier d'erreurs découlant de besoins communicatifs des apprenants qui ne trouvent pas leur " assouvissement » dans leur compétence du moment. Ce sont les besoins communicatifs qui restent le motif premier de la prise de parole soit à l'oral soit à l'écrit et ils poussent à courir le risque de l'erreur malgré les « coûts » au niveau de la correction. 


\section{DE LA TÂCHE SIMULÉE À LA TÂCHE DE LA VIE RÉELLE : TOUJOURS PLUS D’ERREURS ?}

Le réel n'est pas facile à introduire en classe de langue. Il suscite une incrédulité voire une défiance (Ollivier, 2010 : 122) tant de la part des concepteurs des matériaux didactiques que de la part des enseignants. Serait-il vrai que «trop de réalité [...] met(te) les apprenants en danger » ? (Mangenot, Penilla, 2009). Vaudrait-il mieux leur proposer des tâches plausibles au lieu des tâches de la vie réelle ?

Il est encore intéressant de relire le $C E C R$ à ce propos. Nous y retrouverons les « tâches 'cibles' ou de 'répétition' ou 'proches de la vie réelle' qui sont choisies en fonction des besoins de l'apprenant hors de la classe, que ce soit dans les domaines personnel ou public ou en relation à des besoins plus particulièrement professionnels ou éducationnels » (2001: 121) pour ensuite parler des « tâches pédagogiques communicatives [...] assez éloignées de la vie réelle et des besoins des apprenants qui, elles, visent à développer une compétence communicative et sont fondées sur un faire-semblant accepté volontairement 》 (CECR $2001: 121)$.

La classe de langue reste ainsi un lieu de préparation, de par la simulation plus au moins plausible, à son usage réel dans un contexte réel et freine le développement spontané des besoins communicatifs des apprenants qui transgressent, naturellement, ce cadre imposé.

Travailler avec des tâches renvoie à leur double aspect constitutif : d'une part il y a l'aspect situationnel et, de l'autre, l'aspect interactionnel. Le premier ne pose pas aujourd'hui trop de problèmes didactiques, il suffit de proposer de travailler dans des contextes propres au public cible et de viser les genres textuels qui en découlent. Toutes les méthodes de langue actuelles le proposent. Le second aspect, interactionnel, reste encore largement à découvrir dans les pratiques didactiques. Comme le constate Ch. Ollivier (2010 : 122) en citant R. Ellis « l'authenticité interactionnelle (interactional authenticity) doit, elle, permettre une mise en oeuvre de processus communicatifs présents dans la vie réelle », or pour ce dernier la majorité des tâches proposées en classe se limitent à « simulate the kind of communicative acts that learners will experience in real-life contexts $»$.

Marier l'authenticité situationnelle à celle interactionnelle serait donc extrêmement difficile sinon impossible dans la démarche didactique et pourtant les apprenants le demandent car telle est leur expérience de la vie réelle en L1.

Le public avec lequel nous travaillons dans le cadre de la philologie romane pour les débutants est particulièrement exigeant de ce point de vue. Ces étudiants possèdent une conscience aigue de leurs besoins communicatifs ayant une biographie langagière variée et souvent présentant des niveaux de compétence langagière élevés dans d'autres langues. Il n'est donc pas étonnant que cette expérience langagière les prédestine à une pratique communicative qu'ils souhaitent complète et satisfaisante non seulement du point de vue de la norme en L2 mais avant tout du point de vue de 
leurs projets communicationnels auxquels ils tiennent beaucoup et qu'ils essaient de réaliser même en prenant de hauts risques en langue qu'ils ne maîtrisent pas encore suffisamment.

Dans le cadre de l'épreuve de mi-parcours ces étudiants ont passé un test de français comprenant différentes activités visant l'évaluation des compétences récéptives et productives. L'une de ces activités portait sur la production écrite et c'est la source directe des données que nous avons recueillies pour la présente communication.

\section{ECRIRE CE QUE L'ON PEUT OU CE QUE L'ON VEUT?}

L'acte d'écriture est particulièrement complexe en L2. Les chercheurs sont d'accord qu'il est plus coûteux cognitivement en L2 qu'en L1 (Dezutter et alii, 2010 : 159). Les différences principales sont les suivantes :

- on produit moins de mots par unité de temps en L2 qu'en L1;

- les pauses sont plus fréquentes en L2 ;

- les interruptions dans le processus de la transcription sont aussi plus fréquentes ;

- la révision des productions en L2 serait plus longue et plus fastidieuse.

La mémoire de travail est souvent surchargée ce qui fait que les activités de « haut niveau » (organisation du texte, adéquation à la situation de communication...) sont minimisées voire inexistantes et seules les activités de « bas niveau » (aspects formels du code) restent, partiellement, dans le centre d'attention de l'apprenant. Pour le reste ce dernier s'appuie sur ce qu'il a déjà « procéduralisé » donc soit sur la L1 soit sur une langue étrangère qu'il maîtrise mieux que le français.

Exprimer son intention de communication à l'écrit signifie ainsi toute une suite d'opérations mentales dont l'opération principale consiste à associer les mots selon les normes de la contiguïté syntaxique, sémantique et stylistique propres à une langue donnée (Bawej 2008 : 92). Recourir à une langue étrangère pour traduire son intention de communication présuppose une nouvelle conceptualisation de cette intention qui s'inscrit dans un contexte différent de celui de la L1 ou celle mieux connue.

Les langues procèdent à des profilages linguistiques différents de la réalité qui nous entoure. Ces profils sont conventionnels et ne nécessitent plus le passage par une réflexion approfondie, il s'agit souvent des expressions phraséologiques, des collocations, des expressions figées comme proverbes ou dictons. Nous attribuons à la langue étrangère les profils que nous transférons de notre L1 sans nous en rendre compte et c'est là une source de déviances très importante en L2. Avec la notion du profil linguistique, l'on encourage à interroger les langues quels éléments d'un concept sont mis en avant et comment ce même concept est profilé en L1 ou dans une langue mieux connue. C'est cette notion qui est à l'origine de la différence par exemple entre ces deux proverbes : Tout ce qui brille n'est pas or vs Nie wszystko złoto, co się świeci (Florczak, $2010: 54)$. 
Cependant il n'y a pas que les proverbes ou les phraséologismes auxquels il faudrait sensibiliser les apprenants. La présence des structures conventionnelles dans une langue est très importante, elles recouvrent presque $40 \%$ du lexique. Les anglosaxons ont même forgé le terme de "formulaic competence » sensibilisant à l'existence des expressions emmagasinés dans la mémoire de l'usager et fonctionnant comme des entités que l'on ne réduit pas à leurs parties constitutives. Leur manque mène droit vers le transfer négatif de la L1 (Florczak, 2010 : 93).

Enfin, il serait important de démontrer comment une langue à dominante synthétique telle que le polonais découpe conceptuellement la réalité par rapport à une langue analytique comme le français ${ }^{1}$.

C'est avec ces outils de repérage d'erreurs que nous avons procédé à notre étude empirique.

\section{ANALYSE DU CORPUS}

Nous avons analysé 28 copies des étudiants de la I Ìre année « débutant » en philologie romane. Ils ont réalisé deux activités de production écrite lors de l'épreuve de mi-parcours en janvier 2011 dont les consignes étaient les suivantes :

1. Vous avez 40 ans, vous avez un poste à responsabilité dans une entreprise. Vos enfants ont 10 et 5 ans. Une tante vous invite à passer le week-end chez elle pour fêter son anniversaire de mariage. Vous n'avez pas envie d'y aller. Vous êtes fatigué(e) et vous n'aimez pas beaucoup cette personne. Mais vous ne voulez pas être impoli(e). Vous répondez à votre tante pour refuser son invitation. Rédigez la lettre.

2. Vous vous êtes installé(e) dans une nouvelle ville pour vos études. Vous écrivez un e-mail à un(e) ami(e) pour lui parler de votre nouvelle vie. Vous lui expliquez ce que vous faites. Les informations suivantes doivent apparaître dans votre message :

- mieszkanie w centrum, naprzeciwko dworca,

- zajęcia od poniedziałku do czwartku,

- w piątki zwiedzanie miasta,

- wieczorami kino, spotkania z kolegami, odpoczynek w domu,

- ludzie bardzo mili,

- wszyscy mówią po angielsku,

- jest bardzo ciepło ${ }^{2}$.

Rappelons que nous n'avons pas retenu les erreurs relevant de la simple méconnaissance du code linguistique, nous nous sommes uniquement intéressée à des énoncés transgressant la norme sociolinguistique et socioculturelle suite à la méconnaissance des mécanismes décrits ci-dessus.

\footnotetext{
${ }^{1}$ C'est une simplification voulue pour mieux mettre en relief les sources d'erreurs potentielles.

${ }^{2}$ Logement dans le centre, en face de la gare - cours du lundi au jeudi - le vendredi : visites de la ville - le soir : cinéma, sorties avec des copains, repos à la maison - les gens sympathiques - ils parlent tous anglais - il fait chaud.
} 


\subsection{DONNÉES QUANTITATIVES ET QUALITATIVES ${ }^{3}$}

Nous avons examiné 28 copies en repertoriant 67 erreurs entrant dans les catégories délimitées (dans les copies des S6 et S27 il n’y a pas eu d'erreurs de type recherché).

\begin{tabular}{|l|c|l|}
\hline \multicolumn{1}{|c|}{ Type d'erreurs } & Nombre & \multicolumn{1}{c|}{ Remarques } \\
\hline Usage conventionnel & 29 & \\
\hline Profilage & 24 & \\
\hline $\begin{array}{l}\text { Caractère synthétique/analytique } \\
\text { de la langue }\end{array}$ & 14 & $\begin{array}{l}\text { Dont 9 relevant de la } \\
\text { même structure }\end{array}$ \\
\hline Total & 67 & \\
\hline
\end{tabular}

\subsubsection{ERREURS RELEVANT DU TRANSFER DE L'USAGE CONVENTIONNEL EN L1 SUR LA L2 (FORMULAIC COMPETENCE)}

\begin{tabular}{|c|c|c|c|}
\hline Copie & Énoncé erroné & Source présumée & Version corrigée \\
\hline \multirow[t]{2}{*}{ S2 } & $\begin{array}{l}\text { Je voudrais fêter votre } \\
\text { anniversaire, je pense } \\
\text { que c'est incroyable } \\
\text { que vous et l'oncle } \\
\text { Jacques êtes ensemble } \\
\text { tellement longtemps, mais } \\
\text { malheureusement je ne peux } \\
\text { pas. }\end{array}$ & $\begin{array}{l}\text { Chciał(a)bym świętować } \\
\text { waszą rocznicę, myślę, że } \\
\text { to niewiarygodne, że ciocia } \\
\text { i wujek Jacques jesteście } \\
\text { ze sobą już tak długo, ale } \\
\text { niestety nie mogę. }\end{array}$ & $\begin{array}{l}\text { Je voudrais vraiment } \\
\text { pouvoir venir à votre } \\
\text { anniversaire, car je pense } \\
\text { que c'est incroyable que } \\
\text { l'oncle Jacques et vous } \\
\text { soyez ensemble depuis } \\
\text { si longtemps, mais je ne } \\
\text { pourrai malheureusement } \\
\text { pas. }\end{array}$ \\
\hline & $\begin{array}{l}\text { Je n'ai pas eu de vacances } \\
\text { depuis quand Marie avait } 2 \\
\text { ans et elle a maintenant } 5 \text { ! }\end{array}$ & $\begin{array}{l}\text { Nie miałem wakacji odkąd } \\
\text { Marie skończyła } 2 \text { latka, } \\
\text { a teraz ma już } 5 \text { ! }\end{array}$ & $\begin{array}{l}\text { Je n'ai pas eu de vacances } \\
\text { depuis que Marie a eu ses } \\
\text { deux ans... Et elle vient } \\
\text { d'en avoir cinq! }\end{array}$ \\
\hline S3 & $\begin{array}{l}\text { Je suis désolée mais je dois } \\
\text { refuser. }\end{array}$ & $\begin{array}{l}\text { Przykro mi, ale muszę } \\
\text { odmówić. }\end{array}$ & $\begin{array}{l}\text { Je suis désolée, mais je ne } \\
\text { vais pas pouvoir. }\end{array}$ \\
\hline \multirow[t]{2}{*}{ S5 } & $\begin{array}{l}\text { Chaque vendredi je vais } \\
\text { faire des promenades et (de) } \\
\text { sight-seeing avec un guide. }\end{array}$ & $\begin{array}{l}\text { W piątki chodzę na } \\
\text { spacery i na zwiedzanie } \\
\text { z przewodnikiem. }\end{array}$ & $\begin{array}{l}\text { Chaque vendredi je fais du } \\
\text { tourisme avec un guide et je } \\
\text { me promène. }\end{array}$ \\
\hline & $\begin{array}{l}\text { J'ai des rendez-vous avec } \\
\text { mes copins pendant des } \\
\text { après-midi ou je vais au } \\
\text { cinéma ou je reste à la } \\
\text { maison. }\end{array}$ & $\begin{array}{l}\text { Spotykam się z kolegami } \\
\text { popołudniami, albo chodzę } \\
\text { do kina albo zostaję } \\
\text { w domu. }\end{array}$ & $\begin{array}{l}\text { Je rencontre mes copains } \\
\text { certains après-midis, je vais } \\
\text { au cinéma ou bien je reste } \\
\text { à la maison. }\end{array}$ \\
\hline
\end{tabular}

${ }^{3}$ Nous tenons à remercier M. Sébastien Ducourtioux, doctorant à l'Institut d'Etudes Romanes à l'Université de Varsovie, pour son aide précieuse apportée dans les « versions corrigées ». 
suite

\begin{tabular}{|c|c|c|c|}
\hline Copie & Énoncé erroné & Source présumée & Version corrigée \\
\hline S8 & $\begin{array}{l}\text { Je suis vraiment désolée } \\
\text { mais je dois refuser } \\
\text { l'invitation. }\end{array}$ & $\begin{array}{l}\text { Naprawdę jest mi przykro, } \\
\text { ale muszę odmówić. }\end{array}$ & $\begin{array}{l}\text { Je suis vraiment désolée, } \\
\text { mais je ne vais pouvoir } \\
\text { venir. }\end{array}$ \\
\hline \multirow[t]{3}{*}{ S10 } & $\begin{array}{l}\text { Je veux vivre ici tout le } \\
\text { temps. }\end{array}$ & Chcę tu mieszkać na stałe. & $\begin{array}{l}\text { Je veux vivre ici pour } \\
\text { toujours. }\end{array}$ \\
\hline & $\begin{array}{l}\text { Félicitations pour votre } \\
\text { anniversaire mais je ne peux } \\
\text { pas aller. }\end{array}$ & $\begin{array}{l}\text { Gratulacje z okazji rocznicy, } \\
\text { ale nie mogę przyjechać. }\end{array}$ & \begin{tabular}{|l|} 
Félicitations pour votre \\
anniversaire de mariage. Je \\
ne vais malheureusement \\
pas pouvoir venir. \\
\end{tabular} \\
\hline & $\begin{array}{l}\text { Merci pour votre invitation } \\
\text { et tous les meilleurs. }\end{array}$ & $\begin{array}{l}\text { Dziękuję za zaproszenie } \\
\text { i wszystkiego najlepszego. }\end{array}$ & $\begin{array}{l}\text { Merci de votre invitation, } \\
\text { nous vous envoyons tous } \\
\text { nos meilleurs vœux. }\end{array}$ \\
\hline \multirow[t]{3}{*}{$\mathrm{S} 12$} & $\begin{array}{l}\text { Excuse-moi encore une } \\
\text { fois et félicitations pour } \\
\text { l'anniversaire. }\end{array}$ & $\begin{array}{l}\text { Jeszcze raz przepraszam } \\
\text { i gratulacje z okazji } \\
\text { rocznicy. }\end{array}$ & $\begin{array}{l}\text { Excuse-moi encore une fois, } \\
\text { et toutes mes félicitations } \\
\text { pour cet anniversaire. }\end{array}$ \\
\hline & $\begin{array}{l}\text { C'est tout ce que j'ai voulu } \\
\text { t'écrire. J'attends ta réponse. }\end{array}$ & $\begin{array}{l}\text { To wszystko o czym } \\
\text { chciałem Ci napisać. } \\
\text { Czekam na odpowiedź. }\end{array}$ & $\begin{array}{l}\text { Voilà c'est tout pour le } \\
\text { moment. J'attends ta } \\
\text { réponse. }\end{array}$ \\
\hline & $\begin{array}{l}\text { Je dois t'écrire de ma } \\
\text { nouvelle vie. }\end{array}$ & $\begin{array}{l}\text { Muszę Ci opisać moje nowe } \\
\text { życie. }\end{array}$ & $\begin{array}{l}\text { Je dois te parler de ma } \\
\text { nouvelle vie. }\end{array}$ \\
\hline \multirow[t]{2}{*}{ S14 } & $\begin{array}{l}\text { Nous vous souhaitons } \\
\text { tout le meilleur pour votre } \\
\text { anniversaire. }\end{array}$ & $\begin{array}{l}\text { Życzymy wam wszystkiego } \\
\text { najlepszego z okazji } \\
\text { rocznicy. }\end{array}$ & $\begin{array}{l}\text { Tous nos vœux de bonheur } \\
\text { à l'occasion de cet } \\
\text { anniversaire. }\end{array}$ \\
\hline & $\begin{array}{l}\text { Je me rencontre avec des } \\
\text { amis de la faculté. }\end{array}$ & $\begin{array}{l}\text { Spotykam się z kolegami ze } \\
\text { studiów. }\end{array}$ & $\begin{array}{l}\text { Je sors avec des amis de la } \\
\text { faculté (ou : je rencontre des } \\
\text { amis de la faculté) }\end{array}$ \\
\hline S15 & $\begin{array}{l}\text { Nous voulons se rencontrer } \\
\text { l'autre jour pour célébrer } \\
\text { votre anniversaire ! Ecrit } \\
\text { rapidement! }\end{array}$ & $\begin{array}{l}\text { Chcemy spotkać się innego } \\
\text { dnia, żeby uczcić rocznicę. } \\
\text { Odpisz szybko! }\end{array}$ & $\begin{array}{l}\text { Nous voulons vous voir } \\
\text { un autre jour pour célébrer } \\
\text { votre anniversaire, écrivez- } \\
\text { nous rapidement. }\end{array}$ \\
\hline \multirow[t]{3}{*}{ S16 } & $\begin{array}{l}\text { Ma famille et moi te } \\
\text { souhaitent! }\end{array}$ & $\begin{array}{l}\text { Moja rodzina i ja składamy } \\
\text { Ci życzenia! }\end{array}$ & $\begin{array}{l}\text { Ma famille et moi te } \\
\text { souhaitons tout le bonheur } \\
\text { possible! }\end{array}$ \\
\hline & $\begin{array}{l}\text { Encore je te souhaite : } \\
\text { félicitations! }\end{array}$ & \begin{tabular}{|l|} 
Jeszcze raz składam \\
życzenia : gratulacje !
\end{tabular} & $\begin{array}{l}\text { Encore une fois : toutes nos } \\
\text { félicitations! }\end{array}$ \\
\hline & $\begin{array}{l}\text { Tes parents sentaient bien? } \\
\text { J'attends ta réponse. }\end{array}$ & $\begin{array}{l}\text { Twoi rodzice dobrze } \\
\text { się czują? Czekam na } \\
\text { odpowiedź. }\end{array}$ & $\begin{array}{l}\text { Tes parents vont bien? } \\
\text { J'attends ta réponse. }\end{array}$ \\
\hline S17 & $\begin{array}{l}\text { Tout de bonne pour ton } \\
\text { anniversaire. }\end{array}$ & $\begin{array}{l}\text { Wszystkiego dobrego } \\
\text { z okazji rocznicy. }\end{array}$ & $\begin{array}{l}\text { Tous nos meilleurs vœux } \\
\text { pour ton anniversaire. }\end{array}$ \\
\hline $\mathrm{S} 20$ & $\begin{array}{l}\text { Je voudrais venir ce } \\
\text { dimanche mais Nicolas va } \\
\text { revenir de ces vacances ; } \\
\text { je le cherche de l'aéroport } \\
\text { et mon deuxième fils } \\
\text { Hugo a mal à la tête et je } \\
\text { l'emmène à notre médecin. }\end{array}$ & $\begin{array}{l}\text { Chciałabym przyjechać } \\
\text { w niedzielę, ale Nicolas } \\
\text { wraca z wakacji ; jadę po } \\
\text { niego na lotnisku, a mojego } \\
\text { drugiego syna Hugo boli } \\
\text { głowa i zabieram go do } \\
\text { lekarza. }\end{array}$ & $\begin{array}{l}\text { Je voudrais venir ce } \\
\text { dimanche, mais Nicolas va } \\
\text { revenir de ses vacances et je } \\
\text { dois le chercher à l'aéroport. } \\
\text { De plus, mon deuxième } \\
\text { fils Hugo a mal à la tête et } \\
\text { je dois l'emmener chez un } \\
\text { médecin. }\end{array}$ \\
\hline
\end{tabular}


suite

\begin{tabular}{|c|c|c|c|}
\hline Copie & Énoncé erroné & Source présumée & Version corrigée \\
\hline & $\begin{array}{l}\text { Je voudrais vous féliciter } \\
\text { (à toi et à l'oncle) je vous } \\
\text { téléphone lundi. }\end{array}$ & $\begin{array}{l}\text { Chciałabym wam } \\
\text { pogratulować (tobie } \\
\text { i wujkowi), zadzwonię } \\
\text { w poniedziałek. } \\
\end{array}$ & $\begin{array}{l}\text { Je vous félicite tous les } \\
\text { deux. Je vous téléphonerai } \\
\text { lundi. }\end{array}$ \\
\hline \multirow[t]{3}{*}{$\mathrm{S} 22$} & $\begin{array}{l}\text { Toutes nos félicitations } \\
\text { pour son anniversaire de } \\
\text { mariage et nos souhaitons de } \\
\text { bonheur aux son époux. }\end{array}$ & $\begin{array}{l}\text { Najlepsze życzenia z okazji } \\
\text { rocznicy ślubu i życzenia } \\
\text { szczęścia dla małżonków. }\end{array}$ & $\begin{array}{l}\text { Toutes nos félicitations } \\
\text { pour votre anniversaire de } \\
\text { mariage et tous nos vœux de } \\
\text { bonheur. }\end{array}$ \\
\hline & Je ne peux pas aller. & Nie mogę przyjechać. & Je ne peux pas venir. \\
\hline & $\begin{array}{l}\text { Je suis très fatigué aussi et } \\
\text { les enfants doivent étudier } \\
\text { beaucoup pour l'école. }\end{array}$ & $\begin{array}{l}\text { Jestem bardzo zmęczony } \\
\text { a dzieci muszą się dużo } \\
\text { uczyć do szkoły. }\end{array}$ & $\begin{array}{l}\text { Je suis aussi très fatigué et } \\
\text { les enfants ont beaucoup de } \\
\text { choses à faire pour l'école. }\end{array}$ \\
\hline \multirow[t]{2}{*}{$\mathrm{S} 23$} & $\begin{array}{l}\text { Nous sommes très heureux } \\
\text { de recevoir et c'est très poli } \\
\text { de vous. }\end{array}$ & $\begin{array}{l}\text { Jesteśmy szczęśliwi } \\
\text { z otrzymania (?), to bardzo } \\
\text { miło z waszej strony. }\end{array}$ & $\begin{array}{l}\text { Nous sommes très heureux } \\
\text { d'avoir reçu une invitation, } \\
\text { c'est très gentil de votre } \\
\text { part. }\end{array}$ \\
\hline & $\begin{array}{l}\text { Malheureusement nous ne } \\
\text { pouvons pas aller à vous. }\end{array}$ & $\begin{array}{l}\text { Niestety nie możemy do was } \\
\text { przyjechać. }\end{array}$ & $\begin{array}{l}\text { Malheureusement nous ne } \\
\text { pouvons pas venir. }\end{array}$ \\
\hline S24 & $\begin{array}{l}\text { Je regrette, je refuse votre } \\
\text { invitation. }\end{array}$ & \begin{tabular}{|l|} 
Żałuję, ale (muszę) \\
odmówić zaproszenia.
\end{tabular} & $\begin{array}{l}\text { Je regrette, je ne vais pas } \\
\text { pouvoir venir. }\end{array}$ \\
\hline S25 & $\begin{array}{l}\text { Je me rencontre avec les } \\
\text { collègues d'université aussi, } \\
\text { j'ai beaucoup d'amis. }\end{array}$ & $\begin{array}{l}\text { Spotykam się z kolegami } \\
\text { z uniwersytetu, mam też } \\
\text { dużo przyjaciół. }\end{array}$ & $\begin{array}{l}\text { Je sors aussi avec les } \\
\text { collègues de l'université, } \\
\text { j'ai beaucoup d'amis }\end{array}$ \\
\hline S26 & $\begin{array}{l}\text { Je voudrais venir mais je } \\
\text { pense que tu comprends ma } \\
\text { décision. }\end{array}$ & $\begin{array}{l}\text { Chciałabym przyjechać, ale } \\
\text { myślę, że zrozumiesz moją } \\
\text { decyzję. }\end{array}$ & $\begin{array}{l}\text { Je voudrais bien venir, mais } \\
\text { je pense que tu comprends } \\
\text { ma décision (de ne pas le } \\
\text { faire) }\end{array}$ \\
\hline
\end{tabular}

\subsubsection{ERREURS RELEVANT D'UN MAUVAIS PROFILAGE LINGUISTIQUE EN L2}

\begin{tabular}{|c|l|l|l|}
\hline Copie & \multicolumn{1}{|c|}{ Enoncés erronés } & \multicolumn{1}{|c|}{ Version corrigée } & \multicolumn{1}{|c|}{ Profils différents } \\
\hline S1 & $\begin{array}{l}\text { J'adore être ici parce que je } \\
\text { fais un connaissance avec } \\
\text { des gens polis et aimables. }\end{array}$ & $\begin{array}{l}\text { J'adore être ici car je fais } \\
\text { connaissance avec des gens } \\
\text { sympathiques. }\end{array}$ & $\begin{array}{l}\text { Polis/aimable vs } \\
\text { sympathiques }\end{array}$ \\
\hline & $\begin{array}{l}\text { Les gens sont importants } \\
\text { pour moi et je m'amuse } \\
\text { quand je peux sortir au } \\
\text { cinéma avec eux. }\end{array}$ & $\begin{array}{l}\text { Les gens sont importants à mes } \\
\text { yeux et j'aime beaucoup aller au } \\
\text { cinéma avec d'autres personnes. }\end{array}$ & $\begin{array}{l}\text { Aller/sortir, pour moi/à } \\
\text { mes yeux, d'autres } \\
\text { personnes }\end{array}$ \\
\hline S2 & $\begin{array}{l}\text { Mais la chose que j'aime le } \\
\text { plus dans cette ville est la } \\
\text { température. Il est vraiment } \\
\text { chaud. }\end{array}$ & $\begin{array}{l}\text { Mais la chose que j'aime le plus } \\
\text { ici est le climat. Il fait vraiment } \\
\text { chaud. }\end{array}$ & $\begin{array}{l}\text { Température/climat } \\
\text { Il est/il fait }\end{array}$ \\
\hline $\begin{array}{l}\text { l'année prochaine, je } \\
\text { promets. }\end{array}$ & $\begin{array}{l}\text { Je vous promets de venir fêter le } \\
\text { prochain avec vous. }\end{array}$ & $\begin{array}{l}\text { Ordre des éléments, } \\
\text { leur importance }\end{array}$ \\
\hline
\end{tabular}


suite

\begin{tabular}{|c|c|c|c|}
\hline Copie & Enoncés erronés & Version corrigée & Profils différents \\
\hline S4 & $\begin{array}{l}\text { Je voudrais te voir la fois } \\
\text { prochaine, par exemple en } \\
\text { vacances./ }\end{array}$ & $\begin{array}{l}\text { Je voudrais te revoir, pendant les } \\
\text { vacances par exemple. }\end{array}$ & $\begin{array}{l}\text { Voir/revoir, ordre des } \\
\text { éléments. }\end{array}$ \\
\hline S5 & $\begin{array}{l}\text { Encore une fois ma chère } \\
\text { tante Brigitte je regrette que } \\
\text { la situation est comme elle } \\
\text { est, mais c'est la vie. }\end{array}$ & $\begin{array}{l}\text { Je regrette encore une fois que ce } \\
\text { soit ainsi, mais c'est la vie. }\end{array}$ & $\begin{array}{l}\text { Ordre des éléments, } \\
\text { structures employées. }\end{array}$ \\
\hline \multirow[t]{3}{*}{ S7 } & $\begin{array}{l}\text { Tout le jour il est vraiment } \\
\text { chaud à Bordeaux. }\end{array}$ & $\begin{array}{l}\text { Il fait chaud toute la journée à } \\
\text { Bordeaux }\end{array}$ & Ordre des éléments \\
\hline & $\begin{array}{l}\text { C'est la raison pourquoi } \\
\text { j'ai un problème avec mon } \\
\text { voisin qui a un manchot } \\
\text { chez lui. }\end{array}$ & $\begin{array}{l}\text { C'est pourquoi j'ai un problème } \\
\text { avec mon voisin qui a un } \\
\text { manchot chez lui. }\end{array}$ & Structure employée \\
\hline & $\begin{array}{l}\text { Une fois encore je voudrais } \\
\text { te remercier pour ton } \\
\text { invitation. }\end{array}$ & $\begin{array}{l}\text { Je te remercie encore une fois } \\
\text { pour ton invitation. }\end{array}$ & Ordre des éléments \\
\hline \multirow[t]{2}{*}{ S8 } & $\begin{array}{l}\text { Maintenant je travaille dans } \\
\text { une entreprise internationale } \\
\text { au poste de beaucoup de } \\
\text { responsabilité. }\end{array}$ & $\begin{array}{l}\text { Je travaille maintenant dans } \\
\text { une multinationale à un poste à } \\
\text { responsabilité. }\end{array}$ & $\begin{array}{l}\text { Ordre des éléments, } \\
\text { vocabulaire }\end{array}$ \\
\hline & $\begin{array}{l}\text { Mes enfants ont préparé } \\
\text { pour vous un dessin. }\end{array}$ & $\begin{array}{l}\text { Mes enfants ont fait un dessin } \\
\text { pour vous. }\end{array}$ & Préparer/faire \\
\hline \multirow[t]{2}{*}{ S9 } & Mais je dois aussi étudier. & Je dois cependant aussi étudier. & Ordre, mais/cependant \\
\hline & $\begin{array}{l}\text { Ma femme doit rester à la } \\
\text { maison et aider Julie. }\end{array}$ & $\begin{array}{l}\text { Nous devons rester à la maison } \\
\text { pour nous occuper de Julie qui } \\
\text { est malade. }\end{array}$ & $\begin{array}{l}\text { Image de la famille, } \\
\text { aider/s'occuper de }\end{array}$ \\
\hline S10 & $\begin{array}{l}\text { Tu peux arriver quand tu } \\
\text { veux. J'attends. }\end{array}$ & $\begin{array}{l}\text { Tu peux venir quand tu veux, je } \\
\text { t'attends. }\end{array}$ & $\begin{array}{l}\begin{array}{l}\text { Organisation de la } \\
\text { phrase }\end{array} \\
\end{array}$ \\
\hline S11 & $\begin{array}{l}\text { Je t'envoie un petit cadeau } \\
\text { avec mes félicitations. }\end{array}$ & $\begin{array}{l}\text { Je t'envoie mes félicitations et un } \\
\text { petit cadeau. }\end{array}$ & $\begin{array}{l}\begin{array}{l}\text { Organisation de la } \\
\text { phrase }\end{array} \\
\end{array}$ \\
\hline \multirow[t]{3}{*}{$\mathrm{S} 15$} & $\begin{array}{l}\text { Est-ce que vous avez samedi } \\
\text { prochain libre? }\end{array}$ & $\begin{array}{l}\text { Etes-vous libres samedi } \\
\text { prochain? }\end{array}$ & Être/avoir \\
\hline & J'ai le vendredi tout libre! & J'ai mon vendredi libre! & Le/mon \\
\hline & $\begin{array}{l}\text { Il fait très chaud : } 25-30^{\circ} \mathrm{C} \\
\text { tout les temps. J'aime la } \\
\text { natation dans une piscine } \\
\text { ouvert. } \\
\end{array}$ & $\begin{array}{l}\text { Il fait très chaud : } 25-30^{\circ} \mathrm{C} \text { tout } \\
\text { le temps, aussi j'aime nager dans } \\
\text { des piscines ouvertes. }\end{array}$ & $\begin{array}{l}\text { Organisation de la } \\
\text { phrase }\end{array}$ \\
\hline S16 & $\begin{array}{l}\text { Je ne peux pas attendre que } \\
\text { tu vas voir mon appartement } \\
\text { et rencontrer mes amis! }\end{array}$ & $\begin{array}{l}\text { Je suis impatient(e) que tu voies } \\
\text { mon appartement et que tu } \\
\text { rencontres mes amis. }\end{array}$ & $\begin{array}{l}\text { Vocabulaire, exprimer } \\
\text { l'impatience }\end{array}$ \\
\hline S18 & $\begin{array}{l}\text { Nous ne pouvons pas } \\
\text { aller parce que je suis } \\
\text { très occupée dans mon } \\
\text { entreprise. }\end{array}$ & $\begin{array}{l}\text { Nous ne pouvons aller à votre } \\
\text { anniversaire parce que je suis très } \\
\text { occupée dans mon entreprise. }\end{array}$ & Aller/aller à \\
\hline S19 & $\begin{array}{l}\text { Anna, ma femme, n'est pas } \\
\text { là, elle a dû aider sa mère } \\
\text { parce que ma belle-mère est } \\
\text { rentrée à la maison après la } \\
\text { maladie. }\end{array}$ & $\begin{array}{l}\text { Anna, ma femme, n'est pas là : } \\
\text { elle doit s'occuper de sa mère qui } \\
\text { est rentrée à la maison après une } \\
\text { maladie. }\end{array}$ & $\begin{array}{l}\text { Organisation de la } \\
\text { phrase, une/la, }\end{array}$ \\
\hline
\end{tabular}


suite

\begin{tabular}{|c|l|l|l|}
\hline Copie & \multicolumn{1}{|c|}{ Enoncés erronés } & \multicolumn{1}{|c|}{ Version corrigée } & \multicolumn{1}{|c|}{ Profils différents } \\
\hline & $\begin{array}{l}\text { Et le vendredi je marche } \\
\text { beaucoup. }\end{array}$ & Je marche beaucoup le vendredi. & Ordre des éléments \\
\hline S23 & $\begin{array}{l}\text { J'aime regarder les nuages, } \\
\text { ils sont charmants. }\end{array}$ & $\begin{array}{l}\text { J'aime regarder les nuages, ils } \\
\text { sont si jolis. }\end{array}$ & Vocabulaire \\
\hline S27 & $\begin{array}{l}\text { J'étudie beaucoup et j'ai un } \\
\text { temps libre aussi. }\end{array}$ & $\begin{array}{l}\text { J'étudie beaucoup, mais j'ai aussi } \\
\text { du temps libre. }\end{array}$ & Et/mais, un/du \\
\hline
\end{tabular}

\subsubsection{ERREURS RELEVANT DES DIFFÉRENCES DANS LA NATURE SYNTHÉTIQUE/ANALYTIQUE DES LANGUES} POLONAISE ET FRANÇAISE

\begin{tabular}{|c|c|c|c|}
\hline Copie & Enoncé erroné & Version corrigée & Éléments en conflit \\
\hline \multirow[t]{2}{*}{ S1 } & $\begin{array}{l}\text { Je suis directrice donc je dois } \\
\text { être pendant ce RV. }\end{array}$ & $\begin{array}{l}\text { Je suis directrice et je me dois } \\
\text { d'être à ce rendez-vous. }\end{array}$ & $\begin{array}{l}\text { Muszę być na tym } \\
\text { spotkaniu/je me dois } \\
\text { d'être }\end{array}$ \\
\hline & $\begin{array}{l}\text { Aussi Paul est tombé malade } \\
\text { hier et je dois m'occuper de } \\
\text { lui. }\end{array}$ & $\begin{array}{l}\text { De plus, Paul est tombé } \\
\text { malade hier, je dois donc rester } \\
\text { m'occuper de lui. }\end{array}$ & $\begin{array}{l}\text { Muszę się nim zająć/ } \\
\text { je dois donc rester } \\
\text { m'occuper de }\end{array}$ \\
\hline \multirow[t]{2}{*}{ S2 } & Tu dois me visiter bientôt (...) & Tu dois bientôt me rendre visite. & $\begin{array}{l}\text { Odwiedzać/rendre } \\
\text { visite à qqn }\end{array}$ \\
\hline & Visite-moi Margot. & Margot! rends-moi visite! & cf. S2 \\
\hline \multirow[t]{2}{*}{$\mathrm{S} 10$} & Tu dois me visiter. & Tu dois me rendre visite. & cf. S2 \\
\hline & $\begin{array}{l}\text { Malheureusement on ne peut } \\
\text { pas vous visiter ce week-end. } \\
\text { On peut vous visiter l'année } \\
\text { prochaine. }\end{array}$ & $\begin{array}{l}\text { Malheureusement nous ne } \\
\text { pourrons pas vous rendre visite } \\
\text { ce weekend. Peut-être l'année } \\
\text { prochaine? }\end{array}$ & $\begin{array}{l}\text { Odwiedzić, możemy } \\
\text { odwiedzić was/rendre } \\
\text { visite à qqn, peut- } \\
\text { être... }\end{array}$ \\
\hline S13 & $\begin{array}{l}\text { Et si tu me visitais? } \\
\text { J'attends ta réponse. }\end{array}$ & $\begin{array}{l}\text { Et si tu me rendais visite? } \\
\text { J'attends ta réponse. }\end{array}$ & cf. S2 \\
\hline S14 & $\begin{array}{l}\text { Je suis à Madrid et, pour } \\
\text { ce moment, je suis très } \\
\text { contente. }\end{array}$ & $\begin{array}{l}\text { Je suis à Madrid, et pour le } \\
\text { moment j'en suis très contente. }\end{array}$ & $\begin{array}{l}\text { Jak na razie, jestem } \\
\text { zadowolona/pour le } \\
\text { moment; j'en ... } \\
\end{array}$ \\
\hline S16 & Tu dois me visiter! & Tu dois me rendre visite! & cf. S2 \\
\hline \multirow[t]{2}{*}{ S17 } & Tu peux me visiter? & Tu peux me rendre visite? & cf. S2 \\
\hline & $\begin{array}{l}\text { Je vais te visiter le mois } \\
\text { prochain. }\end{array}$ & $\begin{array}{l}\text { Je te rendrai visite le mois } \\
\text { prochain. }\end{array}$ & cf. S2 \\
\hline S22 & $\begin{array}{l}\text { Peut-être tu me visites en } \\
\text { décembre? }\end{array}$ & $\begin{array}{l}\text { Peut-être me rendras-tu visite en } \\
\text { décembre? }\end{array}$ & cf. S2 \\
\hline S24 & $\begin{array}{l}\text { Voudrais-tu me visiter? Je } \\
\text { t'invite! }\end{array}$ & $\begin{array}{l}\text { Voudrais-tu me rendre visite? } \\
\text { Je t'invite! }\end{array}$ & cf. S2 \\
\hline S25 & Tu dois aller me visiter. & Tu dois me rendre visite. & cf. S2 \\
\hline
\end{tabular}




\section{EN GUISE DE CONCLUSION}

Cette modeste analyse, loin d'être exhaustive, démontre une nécessité de refléxion sur les sources des erreurs qui sont autres que le système codique de la langue. Les étudiants font de leur mieux en essayant d'exprimer leurs intentions de communication avec les «moyens du bord » sans se rendre compte du fait que ce qui leur manque c'est un autre découpage du monde qui les entoure à travers d'autres moyens linguistiques.

Comme on peut le voir, il ne s'agit pas de moyens langagiers particulièrement compliqués ou plus difficiles de ce que les apprenants étudient mais de structures plus conventionnelles, profilées conformément au français et reflétant le côté analytique dominant en français (plus d'adverbes, de pronoms, de verbes modaux...).

Néanmoins c'est cette réflexion sur la conceptualisation du monde, différente selon les langues, qui doit précéder tout essai d'entraînement langagier, surtout que ces étudiants ont déjà une expérience en langues et retrouveront facilement les bonnes stratégies d'apprentissage et de bons réflexes. Le rôle de l'enseignant est très important dans ce contexte. C'est lui le premier « conceptualisateur » de la L2, qui devrait décortiquer sa manière de conceptualiser le monde en français devant les étudiants en les encourageant à de pareilles démarches.

En fin de compte, plus la conscience sociolinguistique et socioculturelle augmentera, meilleures seront les productions des étudiants habitués désormais non seulement à chercher comment « le dire en français » mais surtout « comment le penser en français ».

BIBLIOGRAPHIE

Alber, J.-L., Py B. (1986). Vers un modèle exolingue de la communication interculturelle : interparole, coopération et conversation, Études de linguistiaue appliquée, 61, in : Cuq J.-P. (2003). Dictionnaire de didactique du français langue étrangère et seconde, Paris : Cle International.

Bawej, I. (2008). Błą leksykalny jako skutek procesów interferencyjnych. Poradnik metodyczny dla dydaktyków języka niemieckiego, Bydgoszcz : Wydawnictwo Uniwersytetu Kazimierza Wielkiego.

Bouton, Ch.-P. (1974). L'acquisition d'une langue étrangère. Aspects théoriques et pratiques. Conséquences pédagogiques essentielles, Paris : Editions Klincksieck.

Cadre Européen Commun de Référence pour les Langues (2001), Paris : Didier.

Corder, S.P. (1967). The significance of learner's errors, International Review of Applied linguistics, 5(4), in : Cuq J.-P. (2003). Dictionnaire de didactique du français langue étrangère et seconde, Paris : Cle International.

Cuq, J.-P. (2003). Dictionnaire de didactique du français langue étrangère et seconde, Paris : Cle International. 
Dirven, R., Radden, G. (2006). La base cognitive du langage : langue et pensée, in : Linguistique cognitive. Comprendre comment fonctionne le langage, dir. N. Delbecque (pp.17-46), Bruxelles : De Boeck-Duculot.

Dezutter, O., Poirier, V., Bleys, F., Cansigno, Y., Flores, O., Silva, H. (2010). Le rapport à l'écriture en français langue étrangère. Le cas des étudiants universitaires, in : Faire vivre les identités : un parcours en francophonie, dir. J.P. Cuq et P. Chardenet (pp. 157-164). Editions des archives contemporaines et Agence universitaire de la Francophonie.

Florczak, J. (2010). Językoznawcze aspekty modelu ksztaltowania kompetencji języka obcego, Łódź : Wydawnictwo Uniwersytetu Łódzkiego.

Mangenot, F., Penilla F. (2009). Internet, tâches et vie réelle, in : Rosen E. (éd.), La perspective actionnelle et l'approche par les tâches en classe de langue, Recherches et applications 45, in : Ollivier Ch. (2010). Ecriture collaborative en ligne : une approche interactionnelle de la production écrite pour des apprenants acteurs sociaux et motivés, Revue française de linguistique appliquée, XV, 121-137. Marquilló Larruy, M. (2003). L'interprétation de l'erreur, Paris : Cle International/VUEF.

Ollivier, Ch. (2010). Écriture collaborative en ligne : une approche interactionnelle de la production écrite pour des apprenants acteurs sociaux et motivés, Revue française de linguistique appliquée, $\mathrm{XV}, 121-137$.

Porquier, R., Frauenfelder U. (1980). Enseignants et apprenants face à l'erreur, in : Le français dans le monde, 154, in : Marquilló Larruy M. (2003). L'interprétation de l'erreur, Paris : Cle International/VUEF. 